\section{A practical method for accurate coordination between the plastic surgeon and the pathologist: The clockwork technique}

Billur Sezgin ${ }^{1}$, Irem Kapucu ${ }^{1}$, Guliz Yenidunya ${ }^{1}$, Pinar Bulutay ${ }^{2}$, Ayse Armutlu' ${ }^{2}$, Selahattin Ozmen ${ }^{1}$, Reha Yavuzer ${ }^{1}$

Departments of ${ }^{1}$ Plastic, Reconstructive, and Aesthetic Surgery and ${ }^{2}$ Pathology, Koc University School of Medicine, Istanbul, Turkey

Correspondence: Billur Sezgin

Department of Plastic, Reconstructive, and Aesthetic Surgery, Koç University Hospital, Maltepe Mh., Davutpasa cad. No 4, Topkapı, 34010 Zeytinburnu, Istanbul, Turkey

Tel: +90-850-250-8250-20201, Fax:+90-0442-232-5300, E-mail: bsezgin@ku.edu.tr

This article was presented as a poster at the 38th Congress of the Turkish Society of Plastic, Reconstructive and Aesthetic Surgeons on 27-30 October, 2016 in Antalya,Turkey.

Received: 15 Feb $2017 \bullet$ Revised: 28 Mar $2017 \bullet$ Accepted: 28 Mar 2017 pISSN: 2234-6163 • elSSN: 2234-6171

https://doi.org/10.5999/aps.2017.00283 • Arch Plast Surg 2018;45:96-97

Copyright (C) 2018 The Korean Society of Plastic and Reconstructive Surgeons This is an Open Access article distributed under the terms of the Creative Commons Attribution Non-Commercial License (http://creativecommons.org/licenses/by-nc/4.0/) which permits unrestricted non-commercial use, distribution, and reproduction in any medium, provided the original work is properly cited.

\section{Dear Editor:}

Cooperation between the surgeon and the pathologist is essential for the correct diagnosis and treatment of skin tumors [1]. Precise communication between these two specialties allows for the precise evaluation of the tumor borders by the pathologist, which in turn reduces the amount of unnecessary tissue removed, thereby improving functional preservation and the aesthetic outcomes of surgery.

Although not every skin tumor excision requires a high level of interdisciplinary interaction, the location and the type of tumor are key elements that can make such a collaboration necessary. This is especially true for tumors located near critical facial aesthetic subunits such as the eyelids, nostrils, and mouth, and for locally aggressive tumors that spread in unpredictable patterns. Mohs micrographic surgery (MMS) is an alternative to conventional surgery in such cases, and allows tissue preservation to be maximized without compromising adequate surgical excision of the tumor [2]. MMS remains an effective, yet not commonly accessible option, as it can only be performed by pathologically trained surgeons. Nevertheless, regardless of the surgical method, establishing the orientation of the specimen is always critical, and accuracy in this regard can be achieved by various techniques, such as using sutures in different lengths or color codes for each side. Surgeons may use hand-drawn pictures, printed maps of the areas in question, and photographs to guide the patholo- gist. Tissue nicks or poly-angulated drawings can also be used to improve the orientation [3]. These markings are especially useful when determining the need for re-excision; thus, precision is key for minimizing further tissue loss while obtaining tumor-free margins. We present an easily applicable technique that has been developed to increase the precision of surgical margin evaluation in various skin tumors.

The 'clockwork technique' uses a clock face template for the orientation of the specimen. This method ensures accurate coordination between the surgeon excising the tumor and the pathologist who guides the surgeon in re-excision until tumor-free margins are achieved. The first step is to determine the type of the lesion via biopsies if necessary, as the safety margin will be adjusted according to the characteristics of the primary tumor. Lesions involving facial subunits may be an exception, as the required margin may not be planned accordingly in certain critical locations. The second step is to outline the approximate borders of the visually apparent tumor and to draw a safety margin that will serve as the clock face for the orientation of the specimen. Then, the periphery of the circle is numbered through 12 as in a clock (Fig. 1). The hourly markings go through both the excised specimen and the healthy tissue, so that the surgeon knows which part of the excised tissue corresponds to which 'hour.' The lesion is excised as a complete circle and a single suture is placed at a pre-determined hourly interval as a guide if the marking is disrupted. After microscopic examination, the pathologist can state which hour slice corresponds to a tumor-positive margin, and further excision can be carried out only in the area of that hour slice.

Plastic surgeons need to work very meticulously alongside pathol-

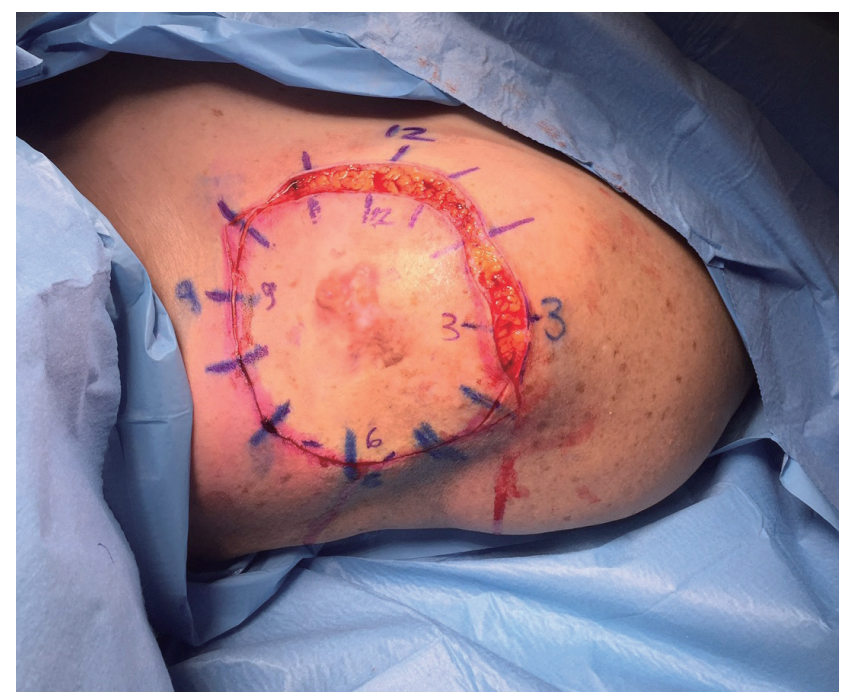

Fig. 1.

A recurrent dermatofibrosarcoma protuberans on the right anterior shoulder of a 45-year-old female patient. A clock face was drawn with $3-\mathrm{cm}$ margins using a surgical marker. Note that the hour markings pass through both the healthy skin and pathological specimen. 
ogists, as every millimeter of skin preserved can be used to achieve better aesthetic and functional outcomes. The clockwork technique can be applied to any type and size of lesion. It is also beneficial for tumors that have unpredictable growth patterns, where it is not possible to agree on a standard optimal safety margin, such as dermatofibrosarcoma protuberans. Moreover, in areas where aesthetic and functional preservation are concerns, it helps to preserve more healthy tissue, as further re-excision can be carried out in only onetwelfth of the excisional border.

In conclusion, this technique is advantageous because it is an easily applicable and fast method that facilitates optimal communication between the surgeon and pathologist regarding specimen borders, allowing precise re-excisions that can be limited to intervals corresponding to one-twelfth of the surgical margin.

\section{Conflict of Interest}

No potential conflict of interest relevant to this article was reported.

\section{References}

1. Thompson JF, Scolyer RA. Cooperation between surgical oncologists and pathologists: a key element of multidisciplinary care for patients with cancer. Pathology 2004;36:496-503.

2. Serra-Guillen C, Llombart B, Nagore E, et al. Mohs micrographic surgery in dermatofibrosarcoma protuberans allows tumour clearance with smaller margins and greater preservation of healthy tissue compared with conventional surgery: a study of 74 primary cases. Br J Dermatol 2015;172:1303-7.

3. Chen TM, Wanitphakdeedecha R, Nguyen TH. Polyangulated specimen and tissue nicks to facilitate orientation and re-excision in Mohs micrographic surgery. Dermatol Surg 2009;35:993-6. 